T. Masuda

Nagoya Math. J.

Vol. 169 (2003), 119-143

\title{
ON THE RATIONAL SOLUTIONS OF $q$-PAINLEVÉ V EQUATION
}

\author{
TETSU MASUDA
}

\begin{abstract}
We give an explicit determinant formula for a class of rational solutions of a $q$-analogue of the Painlevé $V$ equation. The entries of the determinant are given by the continuous $q$-Laguerre polynomials.
\end{abstract}

\section{$\S 1$. Introduction}

Since the introduction of the singularity confinement criterion as the discrete analogue of the Painlevé test [2], a lot of ordinary difference equations have been proposed as discrete Painlevé equations [12], [1]. It is known that the discrete Painlevé equations possess several properties analogous to the continuous ones such as the coalescence cascade, symmetry as the Bäcklund transformations and particular solutions.

Recently, Kajiwara, Noumi and Yamada have proposed a $q$-analogue of the Painlevé IV equation [3], and investigated the structure of symmetry and special solutions of the $q-\mathrm{P}_{\mathrm{IV}}$. It has been shown that the $q-\mathrm{P}_{\mathrm{IV}}$ admits two types of special solutions; one is the special function type solutions, which are expressed in terms of the continuous $q$-Hermite-Weber functions, and another is the rational solutions expressed as the ratio of a $q$-analogue of Okamoto polynomials [11].

In this paper, we consider the symmetric form of $q-\mathrm{P}_{\mathrm{V}}$

$$
\begin{gathered}
\bar{a}_{0}=a_{0}, \quad \bar{a}_{1}=a_{1}, \quad \bar{a}_{2}=a_{2}, \quad \bar{a}_{3}=a_{3} \\
\bar{f}_{0}=a_{0} a_{1} f_{1} \frac{1+a_{2} f_{2}+a_{2} a_{3} f_{2} f_{3}+a_{2} a_{3} a_{0} f_{2} f_{3} f_{0}}{1+a_{0} f_{0}+a_{0} a_{1} f_{0} f_{1}+a_{0} a_{1} a_{2} f_{0} f_{1} f_{2}} \\
\bar{f}_{1}=a_{1} a_{2} f_{2} \frac{1+a_{3} f_{3}+a_{3} a_{0} f_{3} f_{0}+a_{3} a_{0} a_{1} f_{3} f_{0} f_{1}}{1+a_{1} f_{1}+a_{1} a_{2} f_{1} f_{2}+a_{1} a_{2} a_{3} f_{1} f_{2} f_{3}} \\
\bar{f}_{2}=a_{2} a_{3} f_{3} \frac{1+a_{0} f_{0}+a_{0} a_{1} f_{0} f_{1}+a_{0} a_{1} a_{2} f_{0} f_{1} f_{2}}{1+a_{2} f_{2}+a_{2} a_{3} f_{2} f_{3}+a_{2} a_{3} a_{0} f_{2} f_{3} f_{0}}
\end{gathered}
$$

Received July 25, 2001.

2000 Mathematics Subject Classification: 33E17; 34M55. 


$$
\bar{f}_{3}=a_{3} a_{0} f_{0} \frac{1+a_{1} f_{1}+a_{1} a_{2} f_{1} f_{2}+a_{1} a_{2} a_{3} f_{1} f_{2} f_{3}}{1+a_{3} f_{3}+a_{3} a_{0} f_{3} f_{0}+a_{3} a_{0} a_{1} f_{3} f_{0} f_{1}},
$$

with

$$
a_{0} a_{1} a_{2} a_{3}=q^{-1}
$$

where ${ }^{-}$stands for the discrete time evolution. Introducing a variable $c$ by

$$
f_{0} f_{2}=f_{1} f_{3}=c^{-1}
$$

we find that $c$ plays a role of the independent variable,

$$
\bar{c}=q c .
$$

Originally, the equation (1.1) is derived as a subsystem of the discrete dynamical systems associated with the extended affine Weyl group symmetry of type $A_{m-1}^{(1)} \times A_{n-1}^{(1)}$ [4]. In the case of $(m, n)=(2,4)$, by regarding a translation of $\widetilde{W}\left(A_{1}^{(1)}\right)$ as the discrete time evolution ${ }^{-}$, we obtain the system (1.1). The variables $q$ and $c$ are invariant for the action of $\widetilde{W}\left(A_{1}^{(1)} \times A_{3}^{(1)}\right)$ and $\widetilde{W}\left(A_{3}^{(1)}\right)$, respectively. The inverse time evolution of (1.1) is given by

$$
\begin{gathered}
\underline{a_{0}}=a_{0}, \quad \underline{a_{1}}=a_{1}, \quad \underline{a_{2}}=a_{2}, \quad \underline{a_{3}}=a_{3}, \\
\underline{f_{0}}=\frac{f_{3}}{a_{0} a_{1}} \frac{a_{2} a_{1} a_{0}+a_{1} a_{0} f_{2}+a_{0} f_{2} f_{1}+f_{2} f_{1} f_{0}}{a_{0} a_{3} a_{2}+a_{3} a_{2} f_{0}+a_{2} f_{0} f_{3}+f_{0} f_{3} f_{2}}, \\
\underline{f_{1}}=\frac{f_{0}}{a_{1} a_{2}} \frac{a_{3} a_{2} a_{1}+a_{2} a_{1} f_{3}+a_{1} f_{3} f_{2}+f_{3} f_{2} f_{1}}{a_{1} a_{0} a_{3}+a_{0} a_{3} f_{1}+a_{3} f_{1} f_{0}+f_{1} f_{0} f_{3}}, \\
\underline{f_{2}}=\frac{f_{1}}{a_{2} a_{3}} \frac{a_{0} a_{3} a_{2}+a_{3} a_{2} f_{0}+a_{2} f_{0} f_{3}+f_{0} f_{3} f_{2}}{a_{2} a_{1} a_{0}+a_{1} a_{0} f_{2}+a_{0} f_{2} f_{1}+f_{2} f_{1} f_{0}}, \\
\underline{f_{3}}=\frac{f_{0}}{a_{3} a_{0}} \frac{a_{1} a_{0} a_{3}+a_{0} a_{3} f_{1}+a_{3} f_{1} f_{0}+f_{1} f_{0} f_{3}}{a_{3} a_{2} a_{1}+a_{2} a_{1} f_{3}+a_{1} f_{3} f_{2}+f_{3} f_{2} f_{1}} .
\end{gathered}
$$

The reason why we refer to the discrete system (1.1) as the symmetric form of $q-\mathrm{P}_{\mathrm{V}}$ is as follows. By the original construction in [4], it is clear that this equation admits the affine Weyl group symmetry of type $A_{3}^{(1)}$ as the Bäcklund transformation group, which is stated in Section 3 precisely. Moreover, the system (1.1) reduces to the symmetric form of the Painlevé V equation in the continuum limit. We set

$$
q=e^{\frac{\varepsilon^{2}}{2}}, \quad a_{i}=e^{-\frac{\varepsilon^{2}}{2} \alpha_{i}}, \quad f_{i}=-e^{-\varepsilon \varphi_{i}}, \quad c=e^{\varepsilon \gamma},
$$


and define the derivation $\frac{d}{d s}$ by

$$
\frac{d z}{d s}=\lim _{\varepsilon \rightarrow 0} \frac{\bar{z}-z}{\varepsilon},
$$

for a function $z$ in $\alpha_{i}$ and $\varphi_{i}$. Then, we get from (1.1) and (1.4)

$$
\frac{d \varphi_{0}}{d s}=\frac{1}{\gamma}\left[\varphi_{0} \varphi_{2}\left(\varphi_{1}-\varphi_{3}\right)+\left(\frac{1}{2}-\alpha_{2}\right) \varphi_{0}+\alpha_{0} \varphi_{2}\right], \quad \frac{d \gamma}{d s}=\frac{1}{2}
$$

Introducing the variable $t$ and derivation ' as

$$
\gamma=\sqrt{t}, \quad{ }^{\prime}=t \frac{d}{d t}
$$

we have

$$
\begin{gathered}
\alpha_{0}^{\prime}=0, \quad \alpha_{1}^{\prime}=0, \quad \alpha_{2}^{\prime}=0, \quad \alpha_{3}^{\prime}=0, \\
\varphi_{0}^{\prime}=\varphi_{0} \varphi_{2}\left(\varphi_{1}-\varphi_{3}\right)+\left(\frac{1}{2}-\alpha_{2}\right) \varphi_{0}+\alpha_{0} \varphi_{2}, \\
\varphi_{1}^{\prime}=\varphi_{1} \varphi_{3}\left(\varphi_{2}-\varphi_{0}\right)+\left(\frac{1}{2}-\alpha_{3}\right) \varphi_{1}+\alpha_{1} \varphi_{3}, \\
\varphi_{2}^{\prime}=\varphi_{2} \varphi_{0}\left(\varphi_{3}-\varphi_{1}\right)+\left(\frac{1}{2}-\alpha_{0}\right) \varphi_{2}+\alpha_{2} \varphi_{0}, \\
\varphi_{3}^{\prime}=\varphi_{3} \varphi_{1}\left(\varphi_{0}-\varphi_{2}\right)+\left(\frac{1}{2}-\alpha_{1}\right) \varphi_{3}+\alpha_{3} \varphi_{1} .
\end{gathered}
$$

The normalization conditions (1.2) and (1.3) reduce to

$$
\alpha_{0}+\alpha_{1}+\alpha_{2}+\alpha_{3}=1
$$

and

$$
\varphi_{0}+\varphi_{2}=\varphi_{1}+\varphi_{3}=\sqrt{t}
$$

respectively. This differential system (1.10)-(1.12) is nothing but the symmetric form of $\mathrm{P}_{\mathrm{V}}[10]$.

On the other hand, it has been revealed that a family of the rational solutions of $\mathrm{P}_{\mathrm{V}}$, which exists on the barycenters of Weyl chambers, has a determinant formula whose entries are the Laguerre polynomials [8]. This determinant expression is regarded as a specialization of the universal characters [7]. The aim of this paper is to present an explicit determinant formula for a class of the rational solutions of $q-\mathrm{P}_{\mathrm{V}}$. 
This paper is organized as follows. In Section 2, we give the main result of this paper. In Section 3, we describe the affine Weyl group symmetry and derive a set of bilinear equations for the $\tau$-functions of $q-\mathrm{P}_{\mathrm{V}}$. In Section 4 , we construct the rational solutions of $q-\mathrm{P}_{\mathrm{V}}$. Proof of our result is given in Section 5. Section 6 is devoted to some remarks.

\section{$\S 2$. Main result}

Definition 2.1. Let $p_{k}^{(b)}=p_{k}^{(b)}(y \mid q)$ and $q_{k}^{(b)}=q_{k}^{(b)}(y \mid q), k \in \mathbb{Z}$, be two sets of polynomials defined by

$$
\begin{aligned}
& \sum_{k=0}^{\infty} p_{k}^{(b)} \lambda^{k}=\frac{\left(q^{\frac{1}{4}} b \lambda, q^{\frac{3}{4}} b \lambda ; q\right)_{\infty}}{\left(-q^{\frac{1}{4}} x \lambda,-q^{\frac{3}{4}} x^{-1} \lambda ; q\right)_{\infty}}, \quad p_{k}^{(b)}=0 \text { for } k<0, \\
& \sum_{k=0}^{\infty} q_{k}^{(b)} \lambda^{k}=\frac{\left(-q^{\frac{1}{4}} x \lambda,-q^{\frac{3}{4}} x^{-1} \lambda ; q\right)_{\infty}}{\left(q^{\frac{1}{4}} b^{-1} \lambda, q^{\frac{3}{4}} b^{-1} \lambda ; q\right)_{\infty}}, \quad q_{k}^{(b)}=0 \text { for } k<0,
\end{aligned}
$$

with

$$
y=-\frac{1}{2}\left(q^{-1 / 4} x+q^{1 / 4} x^{-1}\right) .
$$

For $m, n \in \mathbb{Z}_{\geq 0}$, we define a family of polynomials $R_{m, n}^{(b)}=R_{m, n}^{(b)}(y \mid q)$ by

$$
R_{m, n}^{(b)}=\left|\begin{array}{cccccccc}
q_{1}^{(b)} & q_{0}^{(b)} & \cdots & q_{-m+2}^{(b)} & q_{-m+1}^{(b)} & \cdots & q_{-m-n+3}^{(b)} & q_{-m-n+2}^{(b)} \\
q_{3}^{(b)} & q_{2}^{(b)} & \cdots & q_{-m+4}^{(b)} & q_{-m+3}^{(b)} & \cdots & q_{-m-n+5}^{(b)} & q_{-m-n+4}^{(b)} \\
\vdots & \vdots & \ddots & \vdots & \vdots & \ddots & \vdots & \vdots \\
q_{2 m-1}^{(b)} & q_{2 m-2}^{(b)} & \cdots & q_{m}^{(b)} & q_{m-1}^{(b)} & \cdots & q_{m-n+1}^{(b)} & q_{m-n}^{(b)} \\
p_{n-m}^{(b)} & p_{n-m+1}^{(b)} & \cdots & p_{n-1}^{(b)} & p_{n}^{(b)} & \cdots & p_{2 n-2}^{(b)} & p_{2 n-1}^{(b)} \\
\vdots & \vdots & \ddots & \vdots & \vdots & \ddots & \vdots & \vdots \\
p_{-n-m+4}^{(b)} & p_{-n-m+5}^{(b)} & \cdots & p_{-n+3}^{(b)} & p_{-n+4}^{(b)} & \cdots & p_{2}^{(b)} & p_{3}^{(b)} \\
p_{-n-m+2}^{(b)} & p_{-n-m+3}^{(b)} & \cdots & p_{-n+1}^{(b)} & p_{-n+2}^{(b)} & \cdots & p_{0}^{(b)} & p_{1}^{(b)}
\end{array}\right| .
$$

For $m, n \in \mathbb{Z}_{<0}$, we define $R_{m, n}^{(b)}$ by

$$
R_{m, n}^{(b)}=(-1)^{m(m+1) / 2} R_{-m-1, n}^{(b)}, \quad R_{m, n}^{(b)}=(-1)^{n(n+1) / 2} R_{m,-n-1}^{(b)} .
$$

Remark 2.2. The polynomials $p_{k}^{(b)}$ and $q_{k}^{(b)}$ are essentially the continuous $q$-Laguerre polynomials $P_{k}^{(\alpha)}(y \mid q)$, which is defined by [6]

$$
\begin{aligned}
& \sum_{k=0}^{\infty} P_{k}^{(\alpha)}(y \mid q) \lambda^{k}=\frac{\left(q^{\alpha+\frac{1}{2}} \lambda, q^{\alpha+1} \lambda ; q\right)_{\infty}}{\left(q^{\frac{1}{2} \alpha+\frac{1}{4}} e^{i \theta} \lambda, q^{\frac{1}{2} \alpha+\frac{1}{4}} e^{-i \theta} \lambda ; q\right)_{\infty}} \\
& P_{k}^{(\alpha)}(y \mid q)=0 \text { for } k<0, \quad y=\cos \theta .
\end{aligned}
$$


In fact, denoting as

$$
L_{k}(y, b \mid q)=P_{k}^{(\alpha)}(y \mid q), \quad b=q^{\frac{1}{2} \alpha+\frac{1}{4}},
$$

we see that $p_{k}^{(b)}$ and $q_{k}^{(b)}$ are expressed as

$$
\begin{aligned}
& p_{k}^{(b)}(y \mid q)=\left(q^{\frac{3}{4}} b^{-1}\right)^{k} L_{k}\left(y, q^{-\frac{1}{4}} b \mid q\right), \\
& q_{k}^{(b)}(y \mid q)=\left(q^{-\frac{3}{4}} b\right)^{k} L_{k}\left(y, q^{\frac{1}{4}} b^{-1} \mid q^{-1}\right),
\end{aligned}
$$

respectively.

Our main result is stated as follows.

THEOREM 2.3. We set

$$
S_{m, n}(x, a)=R_{m, n}^{(b)}(y), \quad y=-\frac{1}{2}\left(q^{-1 / 4} x+q^{1 / 4} x^{-1}\right), \quad b=q^{-\frac{1}{2}(m-n)} a .
$$

Then, for the parameters

$$
\left(a_{0}, a_{1}, a_{2}, a_{3}\right)=\left(q^{n-\frac{1}{2}} a, a^{-1}, q^{-m-\frac{1}{2}} a, q^{m-n} a^{-1}\right),
$$

we have the following rational solutions of $q-\mathrm{P}_{\mathrm{V}}$,

$$
\begin{aligned}
1+ & q^{\frac{1}{2}(2 n-1)} a f_{0}(x, a) \\
& =q^{\frac{1}{2} m}\left(1+q^{-\frac{1}{2}(m-n+1)} a x^{-1}\right) \frac{S_{m, n}(x, a) S_{m-1, n-1}\left(q^{\frac{1}{2}} x, q^{-1} a\right)}{S_{m, n-1}\left(q^{\frac{1}{2}} x, a\right) S_{m-1, n}\left(x, q^{-1} a\right)}, \\
1+ & a^{-1} f_{1}(x, a) \\
& =q^{\frac{1}{2} n}\left(1+q^{\frac{1}{2}(m-n)} a^{-1} x^{-1}\right) \frac{S_{m-1, n}\left(x, q^{-1} a\right) S_{m, n-1}\left(q^{\frac{1}{2}} x, q a\right)}{S_{m, n}\left(q^{\frac{1}{2}} x, a\right) S_{m-1, n-1}(x, a)}, \\
1+ & q^{-\frac{1}{2}(2 m+1)} a f_{2}(x, a) \\
& =q^{-\frac{1}{2} m}\left(1+q^{-\frac{1}{2}(m-n+1)} a x^{-1}\right) \frac{S_{m-1, n-1}(x, a) S_{m, n}\left(q^{\frac{1}{2}} x, q^{-1} a\right)}{S_{m-1, n}\left(q^{\frac{1}{2}} x, q^{-1} a\right) S_{m, n-1}(x, a)}, \\
1+ & q^{m-n} a^{-1} f_{3}(x, a) \\
& =q^{-\frac{1}{2} n}\left(1+q^{\frac{1}{2}(m-n)} a^{-1} x^{-1}\right) \frac{S_{m, n-1}(x, a) S_{m-1, n}\left(q^{\frac{1}{2}} x, a\right)}{S_{m-1, n-1}\left(q^{\frac{1}{2}} x, a\right) S_{m, n}(x, a)},
\end{aligned}
$$


with $x^{2}=c$. Moreover, the above solutions admit the other expressions as

$$
\begin{aligned}
1+ & q^{-\frac{1}{2}(2 n-1)} a^{-1} f_{0}(x, a) \\
& =q^{-\frac{1}{2} n}\left(1+q^{\frac{1}{2}(m-n+1)} a^{-1} x^{-1}\right) \frac{S_{m, n}\left(q^{\frac{1}{2}} x, a\right) S_{m-1, n-1}\left(x, q^{-1} a\right)}{S_{m, n-1}\left(q^{\frac{1}{2}} x, a\right) S_{m-1, n}\left(x, q^{-1} a\right)} \\
1+ & a f_{1}(x, a) \\
& =q^{\frac{1}{2} m}\left(1+q^{-\frac{1}{2}(m-n)} a x^{-1}\right) \frac{S_{m-1, n}\left(q^{\frac{1}{2}} x, q^{-1} a\right) S_{m, n-1}(x, q a)}{S_{m, n}\left(q^{\frac{1}{2}} x, a\right) S_{m-1, n-1}(x, a)} \\
1+ & q^{\frac{1}{2}(2 m+1)} a^{-1} f_{2}(x, a) \\
& =q^{\frac{1}{2} n}\left(1+q^{\frac{1}{2}(m-n+1)} a^{-1} x^{-1}\right) \frac{S_{m-1, n-1}\left(q^{\frac{1}{2}} x, a\right) S_{m, n}\left(x, q^{-1} a\right)}{S_{m-1, n}\left(q^{\frac{1}{2}} x, q^{-1} a\right) S_{m, n-1}(x, a)} \\
1+ & q^{-m+n} a f_{3}(x, a) \\
& =q^{-\frac{1}{2} m}\left(1+q^{-\frac{1}{2}(m-n)} a x^{-1}\right) \frac{S_{m, n-1}\left(q^{\frac{1}{2}} x, a\right) S_{m-1, n}(x, a)}{S_{m-1, n-1}\left(q^{\frac{1}{2}} x, a\right) S_{m, n}(x, a)}
\end{aligned}
$$

Remark 2.4. The rational solutions of $q-\mathrm{P}_{\mathrm{V}}$ in Theorem 2.3 are a $q$ analogue of those to the Painlevé V equation [8]. See Appendix C in detail.

\section{§3. Weyl group symmetry and bilinear relations}

As we mentioned in Section 1, the symmetric form of $q-\mathrm{P}_{\mathrm{V}}(1.1)$ admits the symmetry of the extended affine Weyl group $\widetilde{W}=\left\langle s_{0}, s_{1}, s_{2}, s_{3}, \pi\right\rangle$ of type $A_{3}^{(1)}$ as a group of Bäcklund transformations. The action of $s_{i}$ and $\pi$ on the variables $a_{i}$ and $f_{i}$ is given by

$$
\begin{gathered}
s_{i}\left(a_{j}\right)=a_{j} a_{i}^{-a_{i j}}, \quad \pi\left(a_{j}\right)=a_{j+1}, \\
s_{i}\left(f_{j}\right)=f_{j}\left(\frac{a_{i}+f_{i}}{1+a_{i} f_{i}}\right)^{u_{i j}}, \quad \pi\left(f_{j}\right)=f_{j+1},
\end{gathered}
$$

where $A=\left(a_{i j}\right)_{i, j=0}^{3}$ is the generalized Cartan matrix of type $A_{3}^{(1)}$ and $U=\left(u_{i j}\right)_{i, j=0}^{3}$ is an orientation matrix of the corresponding Dynkin diagram

$$
A=\left(\begin{array}{cccc}
2 & -1 & 0 & -1 \\
-1 & 2 & -1 & 0 \\
0 & -1 & 2 & -1 \\
-1 & 0 & -1 & 2
\end{array}\right), \quad U=\left(\begin{array}{cccc}
0 & 1 & 0 & -1 \\
-1 & 0 & 1 & 0 \\
0 & -1 & 0 & 1 \\
1 & 0 & -1 & 0
\end{array}\right)
$$


and indices are understood as elements of $\mathbb{Z} / 4 \mathbb{Z}$. These transformations commute with the time evolution and satisfy the fundamental relations

$$
\begin{gathered}
s_{i}^{2}=1, \quad s_{i} s_{j}=s_{j} s_{i}(j \neq i, i \pm 1), \quad s_{i} s_{j} s_{i}=s_{j} s_{i} s_{j}(j=i \pm 1), \\
\pi^{4}=1, \quad \pi s_{j}=s_{j+1} \pi .
\end{gathered}
$$

Let us introduce $\tau$-functions $\tau_{i}$ as solutions of the following equations [5],

$$
\overline{\bar{\tau}}_{i}=g_{i} \frac{\bar{\tau}_{i} \bar{\tau}_{i+1}}{\tau_{i+1}}
$$

where $g_{i}$ is given by

$$
g_{i}=1+a_{i+1} f_{i+1}+a_{i+1} a_{i+2} f_{i+1} f_{i+2}+a_{i+1} a_{i+2} a_{i+3} f_{i+1} f_{i+2} f_{i+3} .
$$

The inverse transformations are given as,

$$
\underline{\tau_{i}}=h_{i} \frac{\tau_{i-1} \tau_{i}}{\bar{\tau}_{i-1}}
$$

with

$$
h_{i}=1+\frac{f_{i-1}}{a_{i-1}}+\frac{f_{i-1} f_{i-2}}{a_{i-1} a_{i-2}}+\frac{f_{i-1} f_{i-2} f_{i-3}}{a_{i-1} a_{i-2} a_{i-3}} .
$$

The Bäcklund transformations can be lifted on the $\tau$-functions as follows:

$$
\begin{gathered}
s_{i}\left(\tau_{i}\right)=\left(1+\frac{f_{i}}{a_{i}}\right) \frac{\bar{\tau}_{i-1} \tau_{i+1}}{\bar{\tau}_{i}}, \quad s_{i}\left(\bar{\tau}_{i}\right)=\left(1+a_{i} f_{i}\right) \frac{\bar{\tau}_{i-1} \tau_{i+1}}{\tau_{i}}, \\
s_{i}\left(\tau_{j}\right)=\tau_{j}, \quad s_{i}\left(\bar{\tau}_{j}\right)=\bar{\tau}_{j}, \quad(i \neq j), \\
\pi\left(\tau_{j}\right)=\tau_{j+1}, \quad \pi\left(\bar{\tau}_{j}\right)=\bar{\tau}_{j+1} .
\end{gathered}
$$

The fundamental relations (3.4) are preserved in this lifting. Note that we have the multiplicative formulas

$$
1+\frac{f_{i}}{a_{i}}=\frac{\bar{\tau}_{i} s_{i}\left(\tau_{i}\right)}{\bar{\tau}_{i-1} \tau_{i+1}}, \quad 1+a_{i} f_{i}=\frac{\tau_{i} s_{i}\left(\bar{\tau}_{i}\right)}{\bar{\tau}_{i-1} \tau_{i+1}}
$$

for the independent variables $f_{i}$ in terms of $\tau$-functions. 
Proposition 3.1. We have the following bilinear equations:

$$
\begin{aligned}
& \tau_{0} s_{0} s_{1}\left(\bar{\tau}_{1}\right)=a_{0}^{2} s_{0}\left(\tau_{0}\right) s_{1}\left(\bar{\tau}_{1}\right)+\left(1-a_{0}^{2}\right) \tau_{2} \bar{\tau}_{3}, \\
& \bar{\tau}_{1} s_{1} s_{0}\left(\tau_{0}\right)=a_{1}^{-2} s_{0}\left(\tau_{0}\right) s_{1}\left(\bar{\tau}_{1}\right)+\left(1-a_{1}^{-2}\right) \tau_{2} \bar{\tau}_{3}, \\
& \tau_{1} s_{1} s_{2}\left(\bar{\tau}_{2}\right)=a_{1}^{2} s_{1}\left(\tau_{1}\right) s_{2}\left(\bar{\tau}_{2}\right)+\left(1-a_{1}^{2}\right) \tau_{3} \bar{\tau}_{0}, \\
& \bar{\tau}_{2} s_{2} s_{1}\left(\tau_{1}\right)=a_{2}^{-2} s_{1}\left(\tau_{1}\right) s_{2}\left(\bar{\tau}_{2}\right)+\left(1-a_{2}^{-2}\right) \tau_{3} \bar{\tau}_{0}, \\
& \tau_{2} s_{2} s_{3}\left(\bar{\tau}_{3}\right)=a_{2}^{2} s_{2}\left(\tau_{2}\right) s_{3}\left(\bar{\tau}_{3}\right)+\left(1-a_{2}^{2}\right) \tau_{3} \bar{\tau}_{1}, \\
& \bar{\tau}_{3} s_{3} s_{2}\left(\tau_{2}\right)=a_{3}^{-2} s_{2}\left(\tau_{2}\right) s_{3}\left(\bar{\tau}_{3}\right)+\left(1-a_{3}^{-2}\right) \tau_{0} \bar{\tau}_{1}, \\
& \tau_{3} s_{3} s_{0}\left(\bar{\tau}_{0}\right)=a_{3}^{2} s_{3}\left(\tau_{3}\right) s_{0}\left(\bar{\tau}_{0}\right)+\left(1-a_{3}^{2}\right) \tau_{1} \bar{\tau}_{2}, \\
& \bar{\tau}_{0} s_{0} s_{3}\left(\tau_{3}\right)=a_{0}^{-2} s_{3}\left(\tau_{3}\right) s_{0}\left(\bar{\tau}_{0}\right)+\left(1-a_{0}^{-2}\right) \tau_{1} \bar{\tau}_{2} .
\end{aligned}
$$

Proof. Eliminating $f_{0}$ from (3.9) with $i=0$, we obtain

$$
1-a_{0}^{2} \frac{\bar{\tau}_{0} s_{0}\left(\tau_{0}\right)}{\tau_{0} s_{0}\left(\bar{\tau}_{0}\right)}=\left(1-a_{0}^{2}\right) \frac{\bar{\tau}_{3} \tau_{1}}{\tau_{0} s_{0}\left(\bar{\tau}_{0}\right)} .
$$

From (3.2) and (3.9), we get

$$
\frac{\tau_{1} s_{0} s_{1}\left(\bar{\tau}_{1}\right)}{s_{0}\left(\bar{\tau}_{0}\right) \tau_{2}}=1+a_{0}^{2}\left(\frac{\tau_{1} s_{1}\left(\bar{\tau}_{1}\right)}{\bar{\tau}_{0} \tau_{2}}-1\right) \frac{\bar{\tau}_{0} s_{0}\left(\tau_{0}\right)}{\tau_{0} s_{0}\left(\bar{\tau}_{0}\right)},
$$

which leads to the first equation of (3.11) by using (3.12). The other equations are derived by the similar way.

Let us define the translation operators $T_{i}(i=0,1,2,3)$ by

$$
T_{1}=\pi s_{3} s_{2} s_{1}, \quad T_{2}=s_{1} \pi s_{3} s_{2}, \quad T_{3}=s_{2} s_{1} \pi s_{3}, \quad T_{0}=s_{3} s_{2} s_{1} \pi,
$$

which commute with each other and satisfy $T_{1} T_{2} T_{3} T_{0}=1$. These operators act on parameters $a_{i}$ as

$$
T_{i}\left(a_{i-1}\right)=q^{-1} a_{i-1}, \quad T_{i}\left(a_{i}\right)=q a_{i}, \quad T_{i}\left(a_{j}\right)=a_{j}(j \neq i-1, i) .
$$

In terms of $T_{i}, \tau$-functions in (3.11) are expressed as

$$
\begin{array}{lll}
\tau_{1}=T_{1}\left(\tau_{0}\right), & \tau_{2}=T_{1} T_{2}\left(\tau_{0}\right), & \tau_{3}=T_{0}^{-1}\left(\tau_{0}\right), \\
s_{0}\left(\tau_{0}\right)=T_{0}^{-1} T_{1}\left(\tau_{0}\right), & s_{1}\left(\tau_{1}\right)=T_{2}\left(\tau_{0}\right), & s_{2}\left(\tau_{2}\right)=T_{1} T_{3}\left(\tau_{0}\right), \\
s_{3}\left(\tau_{3}\right)=T_{3}^{-1}\left(\tau_{0}\right), & s_{0} s_{1}\left(\tau_{1}\right)=T_{1} T_{2} T_{0}^{-1}\left(\tau_{0}\right), & s_{1} s_{0}\left(\tau_{0}\right)=T_{2} T_{0}^{-1}\left(\tau_{0}\right), \\
s_{1} s_{2}\left(\tau_{2}\right)=T_{2} T_{3}\left(\tau_{0}\right), & s_{2} s_{1}\left(\tau_{1}\right)=T_{3}\left(\tau_{0}\right), & s_{2} s_{3}\left(\tau_{3}\right)=T_{2}^{-1}\left(\tau_{0}\right), \\
s_{3} s_{2}\left(\tau_{2}\right)=T_{1} T_{0}\left(\tau_{0}\right), & s_{3} s_{0}\left(\tau_{0}\right)=T_{1} T_{3}^{-1}\left(\tau_{0}\right), & s_{0} s_{3}\left(\tau_{3}\right)=T_{1} T_{3}^{-1} T_{0}^{-1}\left(\tau_{0}\right) .
\end{array}
$$


Introducing a notation,

$$
\tau_{l, m, n}=T_{2}^{l} T_{3}^{m} T_{0}^{n}\left(\tau_{0}\right), \quad \bar{\tau}_{l, m, n}=T_{2}^{l} T_{3}^{m} T_{0}^{n}\left(\bar{\tau}_{0}\right), \quad l, m, n \in \mathbb{Z},
$$

we can express the bilinear relations (3.11) as

$$
\begin{gathered}
\tau_{l, m, n} \bar{\tau}_{l, m-1, n-2}=a_{0}^{2} q^{2 n} \tau_{l-1, m-1, n-2} \bar{\tau}_{l+1, m, n} \\
+\left(1-a_{0}^{2} q^{2 n}\right) \tau_{l, m-1, n-1} \bar{\tau}_{l, m, n-1} \\
\bar{\tau}_{l-1, m-1, n-1} \tau_{l+1, m, n-1}=a_{1}^{-2} q^{2 l} \tau_{l-1, m-1, n-2} \bar{\tau}_{l+1, m, n} \\
+\left(1-a_{1}^{-2} q^{2 l}\right) \tau_{l, m-1, n-1} \bar{\tau}_{l, m, n-1}, \\
\tau_{l-1, m-1, n-1} \bar{\tau}_{l+1, m+1, n}=a_{1}^{2} q^{-2 l} \tau_{l+1, m, n} \bar{\tau}_{l-1, m, n-1} \\
\quad+\left(1-a_{1}^{2} q^{-2 l}\right) \tau_{l, m, n-1} \bar{\tau}_{l, m, n}, \\
\bar{\tau}_{l, m-1, n-1} \tau_{l, m+1, n}=a_{2}^{-2} q^{2(-l+m)} \tau_{l+1, m, n} \bar{\tau}_{l-1, m, n-1} \\
\quad+\left(1-a_{2}^{-2} q^{2(-l+m)}\right) \tau_{l, m, n-1} \bar{\tau}_{l, m, n}, \\
\tau_{l, m-1, n-1} \bar{\tau}_{l-1, m, n}=a_{2}^{2} q^{2(l-m)} \tau_{l-1, m, n-1} \bar{\tau}_{l, m-1, n} \\
\quad+\left(1-a_{2}^{2} q^{2(l-m)}\right) \tau_{l, m, n} \bar{\tau}_{l-1, m-1, n-1} \\
\bar{\tau}_{l, m, n-1} \tau_{l-1, m-1, n}=a_{3}^{-2} q^{2(-m+n)} \tau_{l-1, m, n-1} \bar{\tau}_{l, m-1, n} \\
\quad+\left(1-a_{3}^{-2} q^{2(-m+n)}\right) \tau_{l, m, n} \bar{\tau}_{l-1, m-1, n-1} \\
\tau_{l, m, n-1} \bar{\tau}_{l-1, m-2, n-1}=a_{3}^{2} q^{2(m-n)} \tau_{l, m-1, n} \bar{\tau}_{l-1, m-1, n-2} \\
\quad+\left(1-a_{3}^{2} q^{2(m-n)}\right) \tau_{l-1, m-1, n-1} \bar{\tau}_{l, m-1, n-1}, \\
\bar{\tau}_{l, m, n} \tau_{l-1, m-2, n-2}=a_{0}^{-2} q^{-2 n} \tau_{l, m-m, n} \bar{\tau}_{l-1, m-1, n-2} \\
+\left(1-a_{0}^{-2} q^{-2 n}\right) \tau_{l-1, m-1, n-1} \bar{\tau}_{l, m-1, n-1}
\end{gathered}
$$

\section{$\S 4$. Construction of rational solutions}

In this section, we construct a family of rational solutions of $q-\mathrm{P}_{\mathrm{V}}$. Similarly to the continuous case, we consider the fixed points of Dynkin diagram automorphism $\pi^{2}$ to get a seed solution. It is clear that the symmetric form of $q-\mathrm{P}_{\mathrm{V}}(1.1)$ has a particular solution,

$$
\begin{aligned}
\left(a_{0}, a_{1}, a_{2}, a_{3}\right) & =\left(q^{-\frac{1}{2}} a, a^{-1}, q^{-\frac{1}{2}} a, a^{-1}\right), \\
\left(f_{0}, f_{1}, f_{2}, f_{3}\right) & =\left(x^{-1}, x^{-1}, x^{-1}, x^{-1}\right), \\
x^{2} & =c
\end{aligned}
$$


Applying Bäcklund transformations to the seed solution (4.1), we obtain a family of rational solutions of $q-\mathrm{P}_{\mathrm{V}}$.

Calculating $\tau_{l, m, n}$ and $\bar{\tau}_{l, m, n}$ from (3.2), (3.9) and (3.14), and putting as (4.1) and

$$
\tau_{i}=\bar{\tau}_{i}=1
$$

we obtain the $\tau$-functions for the rational solutions of $q-\mathrm{P}_{\mathrm{V}}$. For small $l, m, n$, we observe that $\tau_{l, m, n}$ and $\bar{\tau}_{l, m, n}$ are factorized as the form

$$
\tau_{l, m, n}=c_{k} U_{l, m, n}, \quad \bar{\tau}_{l, m, n}=\tilde{c}_{k} \bar{U}_{l, m, n}, \quad k=m-n-l .
$$

It is possible to guess that $U_{l, m, n}=U_{l, m, n}(x, a)$ are some polynomials in $x^{-1}, a^{ \pm 1}$ and $q^{ \pm \frac{1}{2}}$, and that the factors $c_{k}$ and $\tilde{c}_{k}$ are determined by the recurrence relations

$$
c_{k+1} \tilde{c}_{k-1}=\left(1+q^{\frac{k}{2}} a^{-1} x^{-1}\right) c_{k} \tilde{c}_{k}, \quad \tilde{c}_{k+1} c_{k-1}=\left(1+q^{-\frac{k}{2}} a x^{-1}\right) c_{k} \tilde{c}_{k}
$$

with the initial conditions

$$
c_{0}=c_{1}=1, \quad \tilde{c}_{0}=\tilde{c}_{1}=1
$$

Some examples of them are listed in Appendix A.

Notice that we have

$$
T_{2}^{l} T_{0}^{l}\left(a_{0}, a_{1}, a_{2}, a_{3}\right)=\left(q^{-\frac{1}{2}} \tilde{a}, \tilde{a}^{-1}, q^{-\frac{1}{2}} \tilde{a}, \tilde{a}^{-1}\right), \quad \tilde{a}=q^{l} a, \quad l \in \mathbb{Z},
$$

under the specialization of (4.1). Comparing (4.6) with (4.1), we see that the effect of $T_{2}$ is absorbed by that of $T_{0}^{-1}$ and rescaling of the parameter $a$. Then, we do not need to consider the translation $T_{2}$ for constructing the family of rational solutions of $q-\mathrm{P}_{\mathrm{V}}$, and it is possible to put

$$
U_{l, m, n}(x, a)=U_{0, m, n-l}\left(x, q^{l} a\right)
$$

Now, by (4.1), (4.3) and (4.7), we can rewrite the bilinear relations 
(3.18) in terms of $U_{m, n}=U_{0, m, n}$. We have

$$
\begin{gathered}
U_{m, n} \bar{U}_{m-1, n-2}=a^{2} q^{2 n-1} \mu_{m-n} U_{m-1, n-1}^{--} \bar{U}_{m, n-1}^{++} \\
\quad+\left(1-a^{2} q^{2 n-1}\right) U_{m-1, n-1} \bar{U}_{m, n-1}, \\
\bar{U}_{m-1, n}^{--} U_{m, n-2}^{++}=a^{2} \mu_{m-n} U_{m-1, n-1}^{--} \bar{U}_{m, n-1}^{++} \\
\quad+\left(1-a^{2}\right) U_{m-1, n-1} \bar{U}_{m, n-1}, \\
U_{m-1, n}^{--} \bar{U}_{m+1, n-1}^{++}=a^{-2} \nu_{m-n} U_{m, n-1}^{++} \bar{U}_{m, n}^{-} \\
\quad+\left(1-a^{-2}\right) U_{m, n-1} \bar{U}_{m, n}, \\
\bar{U}_{m-1, n-1} U_{m+1, n}=a^{-2} q^{2 m+1} \nu_{m-n} U_{m, n-1}^{++} \bar{U}_{m, n}^{--} \\
\quad+\left(1-a^{-2} q^{2 m+1}\right) U_{m, n-1} \bar{U}_{m, n}, \\
U_{m-1, n-1} \bar{U}_{m, n+1}^{--}=a^{2} q^{-2 m-1} \mu_{m-n} U_{m, n}^{--} \bar{U}_{m-1, n} \\
\quad+\left(1-a^{2} q^{-2 m-1}\right) U_{m, n} \bar{U}_{m-1, n}^{--}, \\
\bar{U}_{m, n-1} U_{m-1, n+1}^{--}=a^{2} q^{-2(m-n)} \mu_{m-n} U_{m, n}^{--} \bar{U}_{m-1, n} \\
\quad+\left(1-a^{2} q^{-2(m-n)}\right) U_{m, n} \bar{U}_{m-1, n}^{--}, \\
U_{m, n-1} \bar{U}_{m-2, n}^{--}=a^{-2} q^{2(m-n)} \nu_{m-n} U_{m-1, n} \bar{U}_{m-1, n-1}^{--} \\
\quad+\left(1-a^{-2} q^{2(m-n)}\right) U_{m-1, n}^{--} \bar{U}_{m-1, n-1}, \\
\bar{U}_{m, n} U_{m-2, n-1}^{--}=a^{-2} q^{-2 n+1} \nu_{m-n} U_{m-1, n} \bar{U}_{m-1, n-1}^{--} \\
\quad+\left(1-a^{-2} q^{-2 n+1}\right) U_{m-1, n}^{--} \bar{U}_{m-1, n-1},
\end{gathered}
$$

with the initial conditions

$$
U_{-1,-1}=U_{-1,0}=U_{0,-1}=U_{0,0}=1
$$

where we denote as

$$
\begin{aligned}
& \mu_{k}=\left(1+q^{\frac{1}{2}(k+1)} a^{-1} x^{-1}\right)\left(1+q^{\frac{k}{2}} a^{-1} x^{-1}\right), \\
& \nu_{k}=\left(1+q^{-\frac{1}{2}(k-1)} a x^{-1}\right)\left(1+q^{-\frac{k}{2}} a x^{-1}\right),
\end{aligned}
$$

and

$$
U_{m, n}^{ \pm \pm}=U_{m, n}\left(x, q^{ \pm 1} a\right)
$$

Conversely, by solving the bilinear relations (4.8) with (4.9), we can construct the family of rational solutions of $q-\mathrm{P}_{\mathrm{V}}$. Applying $T_{3}^{m} T_{0}^{n}$ to $(3.10)$ and denoting as $T_{3}^{m} T_{0}^{n}\left(f_{i}\right)=f_{i}(x, a)$, we have the following proposition. 
Proposition 4.1. Let $U_{m, n}=U_{m, n}(x, a)(m, n \in \mathbb{Z})$ be polynomials in $x^{-1}, a^{ \pm 1}$ and $q^{ \pm \frac{1}{2}}$ which satisfy the bilinear equations (4.8) with the initial conditions (4.9). Then, $f_{i}(x, a)$ given by

$$
\begin{aligned}
& 1+q^{\frac{1}{2}(2 n-1)} a f_{0}(x, a) \\
& =\left(1+q^{-\frac{1}{2}(m-n+1)} a x^{-1}\right) \frac{U_{m, n}(x, a) U_{m-1, n-1}\left(q^{\frac{1}{2}} x, q^{-1} a\right)}{U_{m, n-1}\left(q^{\frac{1}{2}} x, a\right) U_{m-1, n}\left(x, q^{-1} a\right)} \\
& 1+a^{-1} f_{1}(x, a)=\left(1+q^{\frac{1}{2}(m-n)} a^{-1} x^{-1}\right) \frac{U_{m-1, n}\left(x, q^{-1} a\right) U_{m, n-1}\left(q^{\frac{1}{2}} x, q a\right)}{U_{m, n}\left(q^{\frac{1}{2}} x, a\right) U_{m-1, n-1}(x, a)}, \\
& 1+q^{-\frac{1}{2}(2 m+1)} a f_{2}(x, a) \\
& \quad=\left(1+q^{-\frac{1}{2}(m-n+1)} a x^{-1}\right) \frac{U_{m-1, n-1}(x, a) U_{m, n}\left(q^{\frac{1}{2}} x, q^{-1} a\right)}{U_{m-1, n}\left(q^{\frac{1}{2}} x, q^{-1} a\right) U_{m, n-1}(x, a)}, \\
& 1+q^{m-n} a^{-1} f_{3}(x, a)=\left(1+q^{\frac{1}{2}(m-n)} a^{-1} x^{-1}\right) \frac{U_{m, n-1}(x, a) U_{m-1, n}\left(q^{\frac{1}{2}} x, a\right)}{U_{m-1, n-1}\left(q^{\frac{1}{2}} x, a\right) U_{m, n}(x, a)}
\end{aligned}
$$

solve the $q-\mathrm{P}_{\mathrm{V}}(1.1)$ for the parameters

$$
\left(a_{0}, a_{1}, a_{2}, a_{3}\right)=\left(q^{n-\frac{1}{2}} a, a^{-1}, q^{-m-\frac{1}{2}} a, q^{m-n} a^{-1}\right) .
$$

Moreover, the above solutions admit the other expressions as

$$
\begin{gathered}
1+q^{-\frac{1}{2}(2 n-1)} a^{-1} f_{0}(x, a) \\
=\left(1+q^{\frac{1}{2}(m-n+1)} a^{-1} x^{-1}\right) \frac{U_{m, n}\left(q^{\frac{1}{2}} x, a\right) U_{m-1, n-1}\left(x, q^{-1} a\right)}{U_{m, n-1}\left(q^{\frac{1}{2}} x, a\right) U_{m-1, n}\left(x, q^{-1} a\right)}, \\
1+a f_{1}(x, a)=\left(1+q^{-\frac{1}{2}(m-n)} a x^{-1}\right) \frac{U_{m-1, n}\left(q^{\frac{1}{2}} x, q^{-1} a\right) U_{m, n-1}(x, q a)}{U_{m, n}\left(q^{\frac{1}{2}} x, a\right) U_{m-1, n-1}(x, a)}, \\
1+q^{\frac{1}{2}(2 m+1)} a^{-1} f_{2}(x, a) \\
=\left(1+q^{\frac{1}{2}(m-n+1)} a^{-1} x^{-1}\right) \frac{U_{m-1, n-1}\left(q^{\frac{1}{2}} x, a\right) U_{m, n}\left(x, q^{-1} a\right)}{U_{m-1, n}\left(q^{\frac{1}{2}} x, q^{-1} a\right) U_{m, n-1}(x, a)}, \\
1+q^{-m+n} a f_{3}(x, a)=\left(1+q^{-\frac{1}{2}(m-n)} a x^{-1}\right) \frac{U_{m, n-1}\left(q^{\frac{1}{2}} x, a\right) U_{m-1, n}(x, a)}{U_{m-1, n-1}\left(q^{\frac{1}{2}} x, a\right) U_{m, n}(x, a)} .
\end{gathered}
$$




\section{$\S 5$. Proof of Theorem 2.3}

In this section, we give the proof for Theorem 2.3.

Proposition 5.1. We have

$$
U_{m, n}=(-1)^{\left(\begin{array}{c}
n+1 \\
2
\end{array}\right)} \kappa_{m} \kappa_{n} S_{m, n}
$$

where $S_{m, n}=S_{m, n}(x, a)$ is defined in Theorem 2.3 and $\kappa_{n}$ is the factor determined by

$$
\kappa_{n+1} \bar{\kappa}_{n-1}=q^{-\frac{1}{4}(2 n+1)} x^{-1}\left(1-q^{2 n+1}\right) \kappa_{n} \bar{\kappa}_{n}, \quad \kappa_{0}=\kappa_{-1}=1 .
$$

We notice that $\kappa_{n}$ for $n \geq 0$ is expressed as

$$
\kappa_{n}=q^{-\left(\begin{array}{c}
n+1 \\
3
\end{array}\right)-\frac{1}{4}\left(\begin{array}{c}
n+1 \\
2
\end{array}\right)} x^{-\left(\begin{array}{c}
n+1 \\
2
\end{array}\right)} \prod_{k=1}^{n} \prod_{j=1}^{k}\left(1-q^{2 j-1}\right) .
$$

By substituting (5.1) into (4.12), we find that Theorem 2.3 is a direct consequence of Propositions 4.1 and 5.1. Taking (5.1) and (2.8) into account, we obtain the bilinear relations for $R_{m, n}^{(b)}$.

Proposition 5.2. The following bilinear relations hold:

$$
\begin{aligned}
& -q^{\frac{1}{4}(2 m-2 n-1)} x\left(1-q^{2 n+1}\right) R_{m, n+1}^{+} \bar{R}_{m-1, n-1} \\
& =b^{2} q^{m+n+1} \mu^{+} R_{m-1, n}^{-} \bar{R}_{m, n}^{++}+x^{2}\left(1-b^{2} q^{m+n+1}\right) R_{m-1, n}^{+} \bar{R}_{m, n}, \\
& -q^{\frac{1}{4}(2 m-6 n-3)} x\left(1-q^{2 n+1}\right) \bar{R}_{m-1, n+1} R_{m, n-1}^{+} \\
& \quad=b^{2} q^{m-n} \mu^{+} R_{m-1, n}^{-} \bar{R}_{m, n}^{++}+x^{2}\left(1-b^{2} q^{m-n}\right) R_{m-1, n}^{+} \bar{R}_{m, n}, \\
& q^{\frac{1}{4}(-6 m+2 n-3)} x\left(1-q^{2 m+1}\right) R_{m-1, n}^{-} \bar{R}_{m+1, n-1} \\
& =b^{-2} q^{-m+n} \nu R_{m, n-1}^{+} \bar{R}_{m, n}^{--}+x^{2}\left(1-b^{-2} q^{-m+n}\right) R_{m, n-1}^{-} \bar{R}_{m, n}, \\
& q^{\frac{1}{4}(-2 m+2 n-1)} x\left(1-q^{2 m+1}\right) \bar{R}_{m-1, n-1} R_{m+1, n}^{-} \\
& =b^{-2} q^{m+n+1} \nu R_{m, n-1}^{+} \bar{R}_{m, n}^{--}+x^{2}\left(1-b^{-2} q^{m+n+1}\right) R_{m, n-1}^{-} \bar{R}_{m, n}, \\
& -q^{-\frac{1}{4}(2 m+6 n+3)} x\left(1-q^{2 n+1}\right) R_{m-1, n-1} \bar{R}_{m, n+1}^{-} \\
& =b^{2} q^{-m-n-1} \mu R_{m, n}^{--} \bar{R}_{m-1, n}^{+}+x^{2}\left(1-b^{2} q^{-m-n-1}\right) R_{m, n} \bar{R}_{m-1, n}^{-}, \\
& -q^{-\frac{1}{4}(2 m+2 n+1)} x\left(1-q^{2 n+1}\right) \bar{R}_{m, n-1}^{-} R_{m-1, n+1}
\end{aligned}
$$




$$
\begin{gathered}
\quad=b^{2} q^{-m+n} \mu R_{m, n}^{--} \bar{R}_{m-1, n}^{+}+x^{2}\left(1-b^{2} q^{-m+n}\right) R_{m, n} \bar{R}_{m-1, n}^{-}, \\
q^{-\frac{1}{4}(2 m+2 n+1)} x\left(1-q^{2 m+1}\right) R_{m+1, n-1}^{--} \bar{R}_{m-1, n}^{-} \\
\quad=b^{-2} q^{m-n+2} \nu^{-} R_{m, n} \bar{R}_{m, n-1}^{---}+x^{2}\left(1-b^{-2} q^{m-n+2}\right) R_{m, n}^{--} \bar{R}_{m, n-1}^{-}, \\
q^{-\frac{1}{4}(6 m+2 n+3)} x\left(1-q^{2 m+1}\right) \bar{R}_{m+1, n}^{-} R_{m-1, n-1}^{--} \\
\quad=b^{-2} q^{-m-n+1} \nu^{-} R_{m, n} \bar{R}_{m, n-1}^{---}+x^{2}\left(1-b^{-2} q^{-m-n+1}\right) R_{m, n}^{--} \bar{R}_{m, n-1}^{-},
\end{gathered}
$$

with

$$
\mu=\left(x+b^{-1}\right)\left(x+q^{\frac{1}{2}} b^{-1}\right), \quad \nu=(x+b)\left(x+q^{-\frac{1}{2}} b\right)
$$

where we denote as

$$
X^{\overbrace{ \pm \cdots \pm}^{j}}=X^{\overbrace{ \pm \cdots \pm}^{j}}(b)=X\left(q^{ \pm \frac{j}{2}} b\right)
$$

From the above discussion, now the proof of Theorem 2.3 is reduced to that of Proposition 5.2.

It is possible to reduce the number of bilinear relations to be proved in (5.4) by the following symmetry of $R_{m, n}^{(b)}(y \mid q)$.

LEMma 5.3. We have the relations for $m, n \in \mathbb{Z}_{\geq 0}$

$$
\begin{gathered}
R_{n, m}^{\left(b^{-1}\right)}\left(y \mid q^{-1}\right)=R_{m, n}^{(b)}(y \mid q) \\
R_{n, m}^{\left(b^{-1}\right)}=(-1)^{m(m+1) / 2+n(n+1) / 2} R_{m, n}^{(b)} .
\end{gathered}
$$

Proof. From (2.7), it is easy to see that

$$
q_{k}^{(b)}(y \mid q)=p_{k}^{\left(b^{-1}\right)}\left(y \mid q^{-1}\right)
$$

which leads to the first relation of Lemma 5.3. To verify the second relation, we introduce polynomials $\tilde{q}_{k}^{(b)}=\tilde{q}_{k}^{(b)}(y \mid q)$ by

$$
\sum_{k=0}^{\infty} \tilde{q}_{k}^{(b)} \lambda^{k}=\frac{\left(-q^{\frac{1}{4}} b^{-1} \lambda,-q^{\frac{3}{4}} b^{-1} \lambda ; q\right)_{\infty}}{\left(q^{\frac{1}{4}} x \lambda, q^{\frac{3}{4}} x^{-1} \lambda ; q\right)_{\infty}}, \quad \tilde{q}_{k}^{(b)}=0 \text { for } k<0 .
$$

Comparing the generating functions, we see that each $\tilde{q}_{k}$ is a linear combination of $q_{j}, j=k, k-2, k-4, \ldots$ Therefore we can express $R_{m, n}^{(b)}$ for 
$m, n \in \mathbb{Z}_{\geq 0}$ in terms of $p_{k}$ and $\tilde{q}_{k}$ as

$$
R_{m, n}^{(b)}=\left|\begin{array}{cccccccc}
\tilde{q}_{1}^{(b)} & \tilde{q}_{0}^{(b)} & \cdots & \tilde{q}_{-m+2}^{(b)} & \tilde{q}_{-m+1}^{(b)} & \cdots & \tilde{q}_{-m-n+3}^{(b)} & \tilde{q}_{-m-n+2}^{(b)} \\
\tilde{q}_{3}^{(b)} & \tilde{q}_{2}^{(b)} & \cdots & \tilde{q}_{-m+4}^{(b)} & \tilde{q}_{-m+3}^{(b)} & \cdots & \tilde{q}_{-m-n+5}^{(b)} & \tilde{q}_{-m-n+4}^{(b)} \\
\vdots & \vdots & \ddots & \vdots & \vdots & \ddots & \vdots & \vdots \\
\tilde{q}_{2 m-1}^{(b)} & \tilde{q}_{2 m-2}^{(b)} & \cdots & \tilde{q}_{m}^{(b)} & \tilde{q}_{m-1}^{(b)} & \cdots & \tilde{q}_{m-n+1}^{(b)} & \tilde{q}_{m-n}^{(b)} \\
p_{n-m}^{(b)} & p_{n-m+1}^{(b)} & \cdots & p_{n-1}^{(b)} & p_{n}^{(b)} & \cdots & p_{2 n-2}^{(b)} & p_{2 n-1}^{(b)} \\
\vdots & \vdots & \ddots & \vdots & \vdots & \ddots & \vdots & \vdots \\
p_{-n-m+4}^{(b)} & p_{-n-m+5}^{(b)} & \cdots & p_{-n+3}^{(b)} & p_{-n+4}^{(b)} & \cdots & p_{2}^{(b)} & p_{3}^{(b)} \\
p_{-n-m+2}^{(b)} & p_{-n-m+3}^{(b)} & \cdots & p_{-n+1}^{(b)} & p_{-n+2}^{(b)} & \cdots & p_{0}^{(b)} & p_{1}^{(b)}
\end{array}\right| .
$$

Noticing that $\tilde{q}_{k}$ and $p_{k}$ are related as

$$
\tilde{q}_{k}^{(b)}(y \mid q)=(-1)^{k} p_{k}^{\left(b^{-1}\right)}(y \mid q),
$$

we obtain the second relation of Lemma 5.3 .

From the symmetries of $R_{m, n}^{(b)}(y \mid q)$ described by (2.4) and Lemma 5.3, it is sufficient to prove the first two relations in (5.4) for $m, n \in \mathbb{Z}_{\geq 0}$, which are equivalent to

$$
\begin{aligned}
& -q^{\frac{1}{4}(2 m-2 n-1)} R_{m, n+1}^{+} \bar{R}_{m-1, n-1}+q^{\frac{1}{4}(2 m+2 n+1)} \bar{R}_{m-1, n+1} R_{m, n-1}^{+} \\
& \quad=x R_{m-1, n}^{+} \bar{R}_{m, n} \\
& -q^{\frac{1}{4}(2 m-6 n-3)} x\left(1-q^{2 n+1}\right) \bar{R}_{m-1, n+1} R_{m, n-1}^{+} \\
& \quad=b^{2} q^{m-n} \mu^{+} R_{m-1, n}^{-} \bar{R}_{m, n}^{++}+x^{2}\left(1-b^{2} q^{m-n}\right) R_{m-1, n}^{+} \bar{R}_{m, n}
\end{aligned}
$$

In the following, we show that these bilinear relations are reduced to Jacobi's identity of determinants. Let $D$ be an $(m+n+1) \times(m+n+1)$ determinant and $D\left[\begin{array}{llll}i_{1} & i_{2} & \cdots & i_{k} \\ j_{1} & j_{2} & \cdots & j_{k}\end{array}\right]$ the minor which are obtained by deleting the rows with indices $i_{1}, \ldots, i_{k}$ and the columns with indices $j_{1}, \ldots, j_{k}$. Then we have Jacobi's identity

$$
\begin{aligned}
D & \cdot D\left[\begin{array}{cc}
m & m+1 \\
1 & m+n+1
\end{array}\right] \\
& =D\left[\begin{array}{c}
m \\
1
\end{array}\right] D\left[\begin{array}{c}
m+1 \\
m+n+1
\end{array}\right]-D\left[\begin{array}{c}
m+1 \\
1
\end{array}\right] D\left[\begin{array}{c}
m \\
m+n+1
\end{array}\right] .
\end{aligned}
$$

We first choose proper determinants as $D$ ( $D$ itself should be expressed in terms of $R_{m, n}^{(b)}$ ). Secondly, we construct such formulas that express the minor 
determinants by $R_{m, n}^{(b)}$. Then, Jacobi's identity yields bilinear equations for $R_{m, n}^{(b)}$ which are nothing but (5.12) and (5.13).

We have the following lemmas.

LEMMA 5.4. We set

$$
D \equiv\left|\begin{array}{ccccc}
q^{-\frac{m+n-2}{2}} q^{\frac{1}{4}} x^{-1} q_{1}^{+} & \bar{q}_{1} & \cdots & \bar{q}_{-m-n+3} & \bar{q}_{-m-n+2} \\
q^{-\frac{m+n-4}{2}} q^{\frac{1}{4}} x^{-1} q_{3}^{+} & \bar{q}_{3} & \cdots & \bar{q}_{-m-n+5} & \bar{q}_{-m-n+4} \\
\vdots & \vdots & \ddots & \vdots & \vdots \\
q^{-\frac{n-m}{2}} q^{\frac{1}{4}} x^{-1} q_{2 m-1}^{+} & \bar{q}_{2 m-1} & \cdots & \bar{q}_{m-n+1} & \bar{q}_{m-n} \\
q^{-n} p_{n-m+1}^{+} & \bar{p}_{n-m+2} & \cdots & \bar{p}_{2 n} & \bar{p}_{2 n+1} \\
\vdots & \vdots & \ddots & \vdots & \vdots \\
q^{-1} p_{-n-m+3}^{+} & \bar{p}_{-n-m+4} & \cdots & \bar{p}_{2} & \bar{p}_{3} \\
p_{-n-m+1}^{+} & \bar{p}_{-n-m+2} & \cdots & \bar{p}_{0} & \bar{p}_{1}
\end{array}\right|
$$

Then, we have

$$
\begin{aligned}
& D=q^{\frac{1}{4} m^{2}-\frac{1}{4} n(n+5)} x^{-m} R_{m, n+1}^{+}, \\
& D\left[\begin{array}{c}
c m \\
1
\end{array}\right]=\bar{R}_{m-1, n+1}, \quad D\left[\begin{array}{c}
c m+1 \\
1
\end{array}\right]=\bar{R}_{m, n}, \\
& D\left[\begin{array}{c}
m \\
m+n+1
\end{array}\right]=q^{\frac{1}{4}(m-1)^{2}-\frac{1}{4}(n-1)(n+4)-1} x^{-m+1} R_{m-1, n}^{+}, \\
& D\left[\begin{array}{c}
m+1 \\
m+n+1
\end{array}\right]=q^{\frac{1}{4} m^{2}-\frac{1}{4}(n-2)(n+3)-1} x^{-m} R_{m, n-1}^{+}, \\
& D\left[\begin{array}{cc}
c c m & m+1 \\
1 & m+n+1
\end{array}\right]=\bar{R}_{m-1, n-1} .
\end{aligned}
$$

Lemma 5.5. Define $P_{j, k}^{[-m-n+j]}$ and $Q_{j, k}^{[-m-n+j]}$ by

$$
\begin{aligned}
& P_{j, k}^{[-m-n+j]}=\prod_{i=1}^{m+n-j}\left(q^{-\frac{1}{4}-\frac{i}{2}} x\right) q^{\frac{1}{2}(m+n-j)\left(k-\frac{1}{2}(m+n-j)+\frac{1}{2}\right)} p_{k}^{[-m-n+j]}, \\
& Q_{j, k}^{[-m-n+j]}=q^{-\frac{1}{2}(m+n-j) k} q_{k}^{[-m-n+j]},
\end{aligned}
$$

where we denote

$$
X^{[j]}=X^{[j]}(x, b)=X\left(q^{\frac{j}{2}} x, q^{\frac{j}{2}} b\right) .
$$


Then, setting

$$
D \equiv\left|\begin{array}{ccccc}
\widetilde{Q}_{0,1}^{[-m-n]++} & Q_{1,1}^{[-m-n+1]} & \cdots & Q_{m+n-1,-m-n+3}^{[-1]} & Q_{m+n,-m-n+2}^{[0]} \\
\widetilde{Q}_{0,3}^{[-m-n]++} & Q_{1,3}^{[-m-n+1]} & \cdots & Q_{m+n-1,-m-n+5}^{[-1]} & Q_{n+n,-m-n+4}^{[0]} \\
\vdots & \vdots & \ddots & \vdots & \vdots \\
\widetilde{Q}_{0,2 m-1}^{[-m-n]++} & Q_{1,2 m-1}^{[-m-n+1]} & \cdots & Q_{m+n-1, m-n+1}^{[-1]} & Q_{m+n, m-n}^{[0]} \\
\widehat{P}_{0,2 n}^{[-m-n]++} & P_{1,2 n+1}^{[-m-n+1]} & \cdots & P_{m+n-1,2 n+1}^{[-1]} & P_{m+n, 2 n+1}^{[0]} \\
\vdots & \vdots & \ddots & \vdots & \vdots \\
\widehat{P}_{0,2}^{[-m-n]++} & P_{1,3}^{[-m-n+1]} & \cdots & P_{m+n-1,3}^{[-1]} & P_{m+n, 3}^{[0]} \\
\widehat{P}_{0,0}^{[-m-n]++} & P_{1,1}^{[-m-n+1]} & \cdots & P_{m+n-1,1}^{[-1]} & P_{m+n, 1}^{[0]}
\end{array}\right|,
$$

where

$$
\begin{aligned}
& \widehat{P}_{0,2 k}^{[-m-n]++}=-\frac{P_{0,2 k}^{[-m-n]++}}{1-q^{2 k+1}} \\
& \widetilde{Q}_{0,2 k-1}^{[-m-n]++}=\frac{Q_{0,2 k-1}^{[-m-n]++}}{q^{m+n+1-2 k}\left(1-q^{-m-n+2 k} b^{2}\right)}
\end{aligned}
$$

we have

$$
\begin{aligned}
& D=(-1)^{n+1} \frac{\prod_{j=1}^{m+n} \mu^{[-m-n+j]}}{\left(q^{-\frac{1}{4}} b^{-2} x\right)^{m+n} \prod_{k=0}^{n}\left(1-q^{2 k+1}\right) \prod_{i=1}^{m} q^{m+n+1-2 i}\left(1-q^{-m-n+2 i} b^{2}\right)} R_{m, n}^{++}, \\
& D\left[\begin{array}{c}
m \\
1
\end{array}\right]=R_{m-1, n+1}, \quad D\left[\begin{array}{c}
m+1 \\
1
\end{array}\right]=R_{m, n}, \\
& D\left[\begin{array}{c}
m \\
m+n+1
\end{array}\right]=(-1)^{n+1} x^{n+1} \\
& q^{-\frac{1}{4} m^{2}+\frac{1}{4} n^{2}+\frac{1}{4} m-\frac{1}{2} n-\frac{3}{4}} \prod_{j=1}^{m+n-1} \mu^{[-m-n+j]} \\
& \times \frac{q_{j=1}}{\left(q^{-\frac{1}{4}} b^{-2} x\right)^{m+n-1} \prod_{k=0}^{n}\left(1-q^{2 k+1}\right) \prod_{i=1}^{m-1} q^{m+n+1-2 i}\left(1-q^{-m-n+2 i} b^{2}\right)} \underline{R}_{m-1, n}^{+}, \\
& D\left[\begin{array}{c}
m+1 \\
m+n+1
\end{array}\right]=(-1)^{n} x^{n} \\
& q^{-\frac{1}{4} m^{2}+\frac{1}{4} n^{2}-\frac{1}{4} m-n} \prod_{j=1}^{m+n-1} \mu^{[-m-n+j]} \\
& \times \frac{q}{\left(q^{-\frac{1}{4}} b^{-2} x\right)^{m+n-1} \prod_{k=0}^{n-1}\left(1-q^{2 k+1}\right) \prod_{i=1}^{m} q^{m+n+1-2 i}\left(1-q^{-m-n+2 i} b^{2}\right)} \underline{R}_{m, n-1}^{+} \text {, }
\end{aligned}
$$




$$
D\left[\begin{array}{cc}
m & m+1 \\
1 & m+n+1
\end{array}\right]=q^{-\frac{1}{4} m^{2}+\frac{1}{4} n^{2}+\frac{1}{4} m-\frac{1}{2} n} x^{n} \underline{R}_{m-1, n}^{-} .
$$

It is easy to see that the bilinear relations (5.12) and (5.13) follow immediately from Jacobi's identity (5.14) by using Lemmas 5.4 and 5.5, respectively. We give the proof of Lemmas 5.4 and 5.5 in Appendix B. This completes the proof of our main result Theorem 2.3.

\section{§6. Remarks}

The $q-\mathrm{P}_{\mathrm{V}}$ (1.1) admits the ultra-discrete limit [14]. The limiting procedure is the same as the case of $q-\mathrm{P}_{\mathrm{IV}}$ [4] and preserves the symmetry of the extended affine Weyl group of type $A_{3}^{(1)}$. Moreover, it is observed that $U_{m, n}=U_{m, n}(x, a)$ are polynomials in $x^{-1}, a^{ \pm 1}$ and $q^{ \pm \frac{1}{2}}$ with positive coefficients. Then, the rational solutions of $q-\mathrm{P}_{\mathrm{V}}$ (1.1) in Theorem 2.3 are thought to survive after taking the ultra-discrete limit.

It is known that the special polynomials associated with the rational solutions of the Painlevé equations possess the mysterious combinatorial properties [16], [9], [15]. It is interesting problem to investigate whether the polynomials $U_{m, n}$ admit such properties.

In [4], it has been shown that the $q-\mathrm{P}_{\mathrm{IV}}$ coincides with Sakai's Mul.6 system [13]. As mentioned in Section 1, the $q-\mathrm{P}_{\mathrm{V}}(1.1)$ has $\widetilde{W}\left(A_{1}^{(1)} \times A_{3}^{(1)}\right)$ symmetry by the original construction. On the other hand, Sakai's Mul.5 system [13], which should be also regarded as a $q$-analogue of the Painlevé V equation, admits the symmetry of $\widetilde{W}\left(A_{4}^{(1)}\right)$. It might be an important problem to study the relationship between the equation (1.1) and Sakai's Mul.5 system.

Acknowledgment. The author would like to thank Prof. M. Noumi, Prof. Y. Yamada and Prof. K. Kajiwara for fruitful discussions.

\section{A. Table of $c_{k}, \tilde{c}_{k}$ and $U_{m . n}$}

The polynomials $U_{m, n}(x, a)$.

$$
\begin{aligned}
& U_{0,0}=1 \\
& \begin{aligned}
U_{1,0}= & +q^{\frac{1}{2}} x^{-2}+a^{-1} q^{\frac{1}{2}}\left(1+q^{\frac{1}{2}}\right) x^{-1} \\
U_{2,0}= & +q^{\frac{3}{2}} x^{-6}+a^{-1}\left(1+q^{\frac{1}{2}}\right)\left(1+q+q^{2}\right)\left(x^{-1}+q x^{-5}\right) \\
& \quad+q^{-\frac{1}{2}}\left(1+q+q^{2}\right)\left[1+a^{-2} q^{\frac{3}{2}}\left(1+q^{\frac{1}{2}}\right)^{2}\right]\left(x^{-2}+q^{\frac{1}{2}} x^{-4}\right)
\end{aligned}
\end{aligned}
$$




$$
+a^{-1} q^{\frac{1}{2}}\left(1+q^{\frac{3}{2}}\right)\left[2\left(1+q^{\frac{1}{2}}+q\right)+a^{-2} q^{2}\left(1+q^{\frac{1}{2}}\right)^{2}\right] x^{-3},
$$

$$
\begin{aligned}
U_{0,1}=1 & +a q^{\frac{1}{2}}\left(1+q^{\frac{1}{2}}\right) x^{-1}+q^{\frac{1}{2}} x^{-2} \\
U_{0,2}=1 & +q^{\frac{3}{2}} x^{-6}+a\left(1+q^{\frac{1}{2}}\right)\left(1+q+q^{2}\right)\left(x^{-1}+q x^{-5}\right) \\
& +q^{-\frac{1}{2}}\left(1+q+q^{2}\right)\left[1+a^{2} q^{\frac{3}{2}}\left(1+q^{\frac{1}{2}}\right)^{2}\right]\left(x^{-2}+q^{\frac{1}{2}} x^{-4}\right) \\
& +a q^{\frac{1}{2}}\left(1+q^{\frac{3}{2}}\right)\left[2\left(1+q^{\frac{1}{2}}+q\right)+a^{2} q^{2}\left(1+q^{\frac{1}{2}}\right)^{2}\right] x^{-3}
\end{aligned}
$$

$$
\begin{aligned}
& U_{1,1}=1+q x^{-4}+\left(1+q^{\frac{1}{2}}\right)\left(a+a^{-1}\right)\left(x^{-1}+q^{\frac{1}{2}} x^{-3}\right) \\
& +q^{-\frac{1}{2}}(1+q)\left(1+q^{\frac{1}{2}}+q\right) x^{-2}, \\
& U_{1,2}=1+q^{2} x^{-8}+a^{-1} q^{-\frac{1}{2}}\left(1+q^{\frac{1}{2}}\right)\left[1+a^{2}\left(1+q+q^{2}\right)\right]\left(x^{-1}+q^{\frac{3}{2}} x^{-7}\right) \\
& +q^{-\frac{3}{2}}\left[a^{2} q^{\frac{3}{2}}\left(1+q^{\frac{1}{2}}\right)^{2}\left(1+q+q^{2}\right)\right. \\
& \left.+\left(1+q^{\frac{1}{2}}+q+q^{2}\right)\left(1+q+q^{\frac{3}{2}}+q^{2}\right)\right]\left(x^{-2}+q x^{-6}\right) \\
& +a^{-1} q^{-1}\left(1+q^{\frac{3}{2}}\right)\left[a^{4} q^{2}\left(1+q^{\frac{1}{2}}\right)^{2}+a^{2}\left(1+q+q^{2}\right)\left(2+3 q^{\frac{1}{2}}+2 q\right)\right. \\
& \left.+\left(1+q^{\frac{1}{2}}+q\right)\right]\left(x^{-3}+q^{\frac{1}{2}} x^{-5}\right) \\
& +q^{-1}\left(1+q^{\frac{1}{2}}+q+q^{\frac{3}{2}}+q^{2}\right) \\
& \times\left[a^{2} q^{\frac{1}{2}}\left(1+q^{\frac{1}{2}}\right)(1+q)\left(1+q^{\frac{3}{2}}\right)+2\left(1+q+q^{2}\right)\right] x^{-4}, \\
& U_{2,1}=1+q^{2} x^{-8}+a^{-1} q^{-\frac{1}{2}}\left(1+q^{\frac{1}{2}}\right)\left[a^{2}+\left(1+q+q^{2}\right)\right]\left(x^{-1}+q^{\frac{3}{2}} x^{-7}\right) \\
& +a^{-2} q^{-\frac{3}{2}}\left[a^{2}\left(1+q^{\frac{1}{2}}+q+q^{2}\right)\left(1+q+q^{\frac{3}{2}}+q^{2}\right)\right. \\
& \left.+q^{\frac{3}{2}}\left(1+q^{\frac{1}{2}}\right)^{2}\left(1+q+q^{2}\right)\right]\left(x^{-2}+q x^{-6}\right) \\
& +a^{-3} q^{-1}\left(1+q^{\frac{3}{2}}\right)\left[a^{4}\left(1+q^{\frac{1}{2}}+q\right)+a^{2}\left(1+q+q^{2}\right)\left(2+3 q^{\frac{1}{2}}+2 q\right)\right. \\
& \left.+q^{2}\left(1+q^{\frac{1}{2}}\right)^{2}\right]\left(x^{-3}+q^{\frac{1}{2}} x^{-5}\right) \\
& +a^{-2} q^{-1}\left(1+q^{\frac{1}{2}}+q+q^{\frac{3}{2}}+q^{2}\right) \\
& \times\left[2 a^{2}\left(1+q+q^{2}\right)+q^{\frac{1}{2}}\left(1+q^{\frac{1}{2}}\right)(1+q)\left(1+q^{\frac{3}{2}}\right)\right] x^{-4} .
\end{aligned}
$$


The factor $c_{k}$ and $\tilde{c_{k}}$.

$$
\begin{aligned}
c_{0}=1, \quad & c_{1}=1, \\
c_{2}=1+ & q^{\frac{1}{2}} a^{-1} x^{-1}, \\
c_{3}= & \left(1+q^{\frac{1}{2}} a^{-1} x^{-1}\right)\left(1+q a^{-1} x^{-1}\right)\left(1+q^{-\frac{1}{2}} a x^{-1}\right), \\
c_{4}= & \left(1+q^{\frac{1}{2}} a^{-1} x^{-1}\right)^{2}\left(1+q a^{-1} x^{-1}\right)\left(1+q^{\frac{3}{2}} a^{-1} x^{-1}\right)\left(1+q^{-\frac{1}{2}} a x^{-1}\right) \\
& \quad \times\left(1+q^{-1} a x^{-1}\right), \\
c_{5}=(1+ & \left.q^{\frac{1}{2}} a^{-1} x^{-1}\right)^{2}\left(1+q a^{-1} x^{-1}\right)^{2}\left(1+q^{\frac{3}{2}} a^{-1} x^{-1}\right)\left(1+q^{2} a^{-1} x^{-1}\right) \\
& \quad \times\left(1+q^{-\frac{1}{2}} a x^{-1}\right)^{2}\left(1+q^{-1} a x^{-1}\right)\left(1+q^{-\frac{3}{2}} a x^{-1}\right), \\
\tilde{c}_{0}=1, \quad & \tilde{c}_{1}=1, \\
\tilde{c}_{2}=1+ & q^{-\frac{1}{2}} a x^{-1}, \\
\tilde{c}_{3}=(1+ & \left.q^{-\frac{1}{2}} a x^{-1}\right)\left(1+q^{-1} a x^{-1}\right)\left(1+q^{\frac{1}{2}} a^{-1} x^{-1}\right), \\
\tilde{c}_{4}= & \left(1+q^{-\frac{1}{2}} a x^{-1}\right)^{2}\left(1+q^{-1} a x^{-1}\right)\left(1+q^{-\frac{3}{2}} a x^{-1}\right)\left(1+q^{\frac{1}{2}} a^{-1} x^{-1}\right) \\
& \quad \times\left(1+q a^{-1} x^{-1}\right), \\
\tilde{c}_{5}= & \left(1+q^{-\frac{1}{2}} a x^{-1}\right)^{2}\left(1+q^{-1} a x^{-1}\right)^{2}\left(1+q^{-\frac{3}{2}} a x^{-1}\right)\left(1+q^{-1} a x^{-2}\right) \\
& \quad \times\left(1+q^{\frac{1}{2}} a^{-1} x^{-1}\right)^{2}\left(1+q a^{-1} x^{-1}\right)\left(1+q^{\frac{3}{2}} a^{-1} x^{-1}\right) .
\end{aligned}
$$

\section{B. Proof of Lemmas 5.4 and $\mathbf{5 . 5}$}

We first note that the following contiguity relations hold,

$$
\begin{aligned}
& p_{k}^{+}-q^{\frac{k}{2}} \bar{p}_{k}=-q^{\frac{1}{4}} x p_{k-1}^{+}, \quad q_{k}^{+}-q^{-\frac{k}{2}} \bar{q}_{k}=-q^{-\frac{1}{4}} x^{-1} q_{k-1}^{+}, \\
& p_{k}-q^{\frac{k}{2}} \underline{p}_{k}^{-}=-q^{\frac{3}{4}} x^{-1} p_{k-1}, \quad q_{k}-q^{-\frac{k}{2}} \underline{q}_{k}^{-}=-q^{-\frac{3}{4}} x q_{k-1},
\end{aligned}
$$

and

$$
\begin{aligned}
\left(1-q^{k+1}\right) p_{k+1} & =q^{\frac{k}{2}+\frac{1}{4}} b x\left(b-b^{-1}\right) \underline{p}_{k}^{+}-q^{\frac{1}{4}} b^{2} x^{-1} \mu p_{k}^{++}, \\
\left(1-q^{k+1} b^{2}\right) q_{k+1} & =q^{\frac{1}{2}(k+1)} b\left(b^{-1}-b\right) \underline{q}_{k+1}^{+}+q^{k+\frac{1}{4}} b^{2} x^{-1} \mu q_{k}^{++},
\end{aligned}
$$

which are easily derived from (2.1). 
Let us prove Lemma 5.4. Noticing that $p_{1}=1$ and $p_{k}=0$ for $k<0$, we see that $R_{m, n}$ can be rewritten as

$$
R_{m, n}=\left|\begin{array}{cccccc}
q_{1} & q_{0} & \cdots & q_{-m-n+3} & q_{-m-n+2} & q_{-m-n+1} \\
q_{3} & q_{2} & \cdots & q_{-m-n+5} & q_{-m-n+4} & q_{-m-n+3} \\
\vdots & \vdots & \ddots & \vdots & \vdots & \vdots \\
q_{2 m-1} & q_{2 m-2} & \cdots & q_{m-n+1} & q_{m-n} & q_{m-n-1} \\
p_{n-m} & p_{n-m+1} & \cdots & p_{2 n-2} & p_{2 n-1} & p_{2 n} \\
\vdots & \vdots & \ddots & \vdots & \vdots & \vdots \\
p_{-n-m+4} & p_{-n-m+5} & \cdots & p_{2} & p_{3} & p_{4} \\
p_{-n-m+2} & p_{-n-m+3} & \cdots & p_{0} & p_{1} & p_{2} \\
p_{-n-m} & p_{-n-m+1} & \cdots & p_{-2} & p_{-1} & p_{0}
\end{array}\right| .
$$

Adding the $(j-1)$-th column multiplied by $q^{\frac{1}{4}} x$ to the $j$-th column of $R_{m, n}^{+}$ for $j=m+n, m+n-1, \ldots, 2$ and using (B.1), we get

$$
\begin{aligned}
R_{m, n}^{+}=q^{-\frac{1}{4} m^{2}+\frac{1}{4}(n-1)(n+4)} x^{m} & \\
& \times\left|\begin{array}{ccccc}
q^{-\frac{m+n-3}{2}} q^{\frac{1}{4}} x^{-1} q_{1}^{+} & \bar{q}_{1} & \cdots & \bar{q}_{-m-n+4} & \bar{q}_{-m-n+3} \\
q^{-\frac{m+n-5}{2}} q^{\frac{1}{4}} x^{-1} q_{3}^{+} & \bar{q}_{3} & \cdots & \bar{q}_{-m-n+6} & \bar{q}_{-m-n+5} \\
\vdots & \vdots & \ddots & \vdots & \vdots \\
q^{-\frac{n-m-1}{2}} q^{\frac{1}{4}} x^{-1} q_{2 m-1}^{+} & \bar{q}_{2 m-1} & \cdots & \bar{q}_{m-n+2} & \bar{q}_{m-n+1} \\
q^{-n+1} p_{n-m}^{+} & \bar{p}_{n-m+1} & \cdots & \bar{p}_{2 n-2} & \bar{p}_{2 n-1} \\
\vdots & \vdots & \ddots & \vdots & \vdots \\
q^{-1} p_{-n-m+4}^{+} & \bar{p}_{-n-m+5} & \cdots & \bar{p}_{2} & \bar{p}_{3} \\
p_{-n-m+2}^{+} & \bar{p}_{-n-m+3} & \cdots & \bar{p}_{0} & \bar{p}_{1}
\end{array}\right| .
\end{aligned}
$$

From (B.4) and (B.5), we obtain Lemma 5.4.

We next prove Lemma 5.5. Adding the $(i+1)$-th column multiplied by $q^{\frac{1}{4}-(m+n-j)} x$ to the $i$-th column of $R_{m, n}$ for $i=1,2, \ldots, j, j=m+n-$ $1, m+n-2, \ldots, 1$ and using (B.2), we get

$$
R_{m, n}=\left|\begin{array}{ccccc}
Q_{1,1}^{[-m-n+1]} & Q_{2,0}^{[-m-n+2]} & \cdots & Q_{m+n-1,-m-n+3}^{[-1]} & Q_{m+n,-m-n+2}^{[0]} \\
Q_{1,3}^{[-m-n+1]} & Q_{2,2}^{[-m-n+2]} & \cdots & Q_{m+n-1,-m-n+5}^{[-1]} & Q_{n+n,-m-n+4}^{[0]} \\
\vdots & \vdots & \ddots & \vdots & \vdots \\
Q_{1,2 m-1}^{[-m-n+1]} & Q_{2,2 m-2}^{[-m-n+2]} & \cdots & Q_{m+n-1, m-n+1}^{[-1]} & Q_{m+n, m-n}^{[0]} \\
P_{1,2 n-1}^{[-m-n+1]} & P_{2,2 n-1}^{[-m-n+2]} & \cdots & P_{m+n-1,2 n-1}^{[-1]} & P_{m+n, 2 n-1}^{[0]} \\
\vdots & \vdots & \ddots & \vdots & \vdots \\
P_{1,3}^{[-m-n+1]} & P_{2,3}^{[-m-n+2]} & \cdots & P_{m+n-1,3}^{[-1]} & P_{m+n, 3}^{[0]} \\
P_{1,1}^{[-m-n+1]} & P_{2,1}^{[-m-n+2]} & \cdots & P_{m+n-1,1}^{[-1]} & P_{m+n, 1}^{[0]}
\end{array}\right|
$$

Noticing that $p_{1}=1$ and $p_{k}=0$ for $k<0$, we see that $R_{m, n}$ can be 
rewritten as

$$
R_{m, n}=\left|\begin{array}{ccccc}
Q_{0,1}^{[-m-n]} & Q_{1,0}^{[-m-n+1]} & \cdots & Q_{m+n-1,-m-n+2}^{[-1]} & Q_{m+n,-m-n+1}^{[0]} \\
Q_{0,3}^{[-m-n]} & Q_{1,2}^{[-m-n+1]} & \cdots & Q_{m+n-1,-m-n+4}^{[-1]} & Q_{n+n,-m-n+3}^{[0]} \\
\vdots & \vdots & \ddots & \vdots & \vdots \\
Q_{0,2 m-1}^{[-m-n]} & Q_{1,2 m-2}^{[-m-n+1]} & \cdots & Q_{m+n-1, m-n}^{[-1]} & Q_{m+n, m-n-1}^{[0]} \\
P_{0,2 n}^{[-m-n]} & P_{1,2 n}^{[-m+n+1]} & \ldots & P_{m+n-1,2 n}^{[-1]} & P_{m+n, 2 n}^{[0]} \\
\vdots & \vdots & \ddots & \vdots & \vdots \\
P_{0,2}^{[-m-n]} & P_{1,2}^{[-m-n+1]} & \cdots & P_{m+n-1,2}^{[-1]} & P_{m+n, 2}^{[0]} \\
P_{0,0}^{[-m-n]} & P_{1,0}^{[-m-n+1]} & \ldots & P_{m+n-1,0}^{[-1]} & P_{m+n, 0}^{[0]}
\end{array}\right|
$$

Then, adding the $j$-th column multiplied $\frac{q^{\frac{3}{4}+m+n-j} b^{-2} x\left(1-q^{-m-n+j} b^{2}\right)}{\mu^{[-m-n+j]}}$ to $(j+1)$-th column of $R_{m, n}^{++}$for $j=m+n, m+n-1, \ldots, 1$ and using (B.3), we obtain

$$
\begin{aligned}
& R_{m, n}^{++}=(-1)^{n+1} \frac{\left(q^{-\frac{1}{4}} b^{-2} x\right)^{m+n} \prod_{k=0}^{n}\left(1-q^{2 k+1}\right) \prod_{i=1}^{m} q^{m+n+1-2 i}\left(1-q^{-m-n+2 i} b^{2}\right)}{\prod_{j=1}^{m+n} \mu^{[-m-n+j]}} \\
& \times\left|\begin{array}{ccccc}
\widetilde{Q}_{0,1}^{[-m-n]++} & Q_{1,1}^{[-m-n+1]} & \cdots & Q_{m+1}^{[-1]} & \\
\widetilde{Q}_{0,3}^{[-m-n]++}+ & Q_{1,3}^{[-m-n+1]} & \cdots & Q_{m+n-1,-m-n+3}^{[-1]} & Q_{m+n,-m-n+2}^{[0]} \\
\vdots & \vdots & \ddots & \vdots & Q_{n+n,-m-n+4}^{[0]} \\
\widetilde{Q}_{0,2 m-1}^{[-m-n]++} & Q_{1,2 m-1}^{[-m-n+1]} & \cdots & Q_{m+n-1, m-n+1}^{[-1]} & Q_{m+n, m-n}^{[0]} \\
\widehat{P}_{0,2 n}^{[-m-n]++} & P_{1,2 n+1}^{[-m-n+1]} & \cdots & P_{m+n-1,2 n+1}^{[-1]} & P_{m+n, 2 n+1}^{[0]} \\
\vdots & \vdots & \ddots & \vdots & \vdots \\
\widehat{P}_{0,2}^{[-m-n]++} & P_{1,3}^{[-m-n+1]} & \cdots & P_{m+n-1,3}^{[-1]} & P_{m+n, 3}^{[0]} \\
\widehat{P}_{0,0}^{[-m-n]++} & P_{1,1}^{[-m-n+1]} & \cdots & P_{m+n-1,1}^{[-1]} & P_{m+n, 1}^{[0]}
\end{array}\right|,
\end{aligned}
$$

where we use the relations

$$
\begin{aligned}
& \underline{P}_{j, k}^{[-m-n+j+1]-}=q^{-\frac{k}{2}+\frac{1}{4}+\frac{1}{2}(m+n-j)} x^{-1} P_{j, k}^{[-m-n+j]}, \\
& \underline{Q}_{j, k}^{[-m-n+j+1]-}=q^{\frac{k}{2}} Q_{j, k}^{[-m-n+j]}
\end{aligned}
$$

Lemma 5.5 follows from (B.6), (B.7) and (B.8).

\section{Continuum limit to the rational solutions of $\mathrm{P}_{\mathrm{V}}$}

We consider the continuum limit of the rational solutions of $q-\mathrm{P}_{\mathrm{V}}$ to those of the Painlevé $\mathrm{V}$ equation. In the previous paper [8], we have presented a determinant formula for the rational solutions of $\mathrm{P}_{\mathrm{V}}$. 
Proposition C.1. Let $\hat{p}_{k}^{(r)}=\hat{p}_{k}^{(r)}(z)$ and $\hat{q}_{k}^{(r)}=\hat{q}_{k}^{(r)}(z)$ be polynomials defined by

$$
\hat{p}_{k}^{(r)}(z)=L_{k}^{(r-1)}(z), \quad \hat{q}_{k}^{(r)}(z)=L_{k}^{(r-1)}(-z),
$$

respectively, where $L_{k}^{(r)}(z)$ are the Laguerre polynomials. For $m, n \in \mathbb{Z}_{\geq 0}$, we define a family of polynomials $\widehat{R}_{m, n}^{(r)}=\widehat{R}_{m, n}^{(r)}(z)$ by

$$
\widehat{R}_{m, n}^{(r)}(z)=\left|\begin{array}{cccccccc}
\hat{q}_{1}^{(r)} & \hat{q}_{0}^{(r)} & \cdots & \hat{q}_{-m+2}^{(r)} & \hat{q}_{-m+1}^{(r)} & \cdots & \hat{q}_{-m-n+3}^{(r)} & \hat{q}_{-m-n+2}^{(r)} \\
\hat{q}_{3}^{(r)} & \hat{q}_{2}^{(r)} & \cdots & \hat{q}_{-m+4}^{(r)} & \hat{q}_{-m+3}^{(r)} & \cdots & \hat{q}_{-m-n+5}^{(r)} & \hat{q}_{-m-n+4}^{(r)} \\
\vdots & \vdots & \ddots & \vdots & \vdots & \ddots & \vdots & \vdots \\
\hat{q}_{2 m-1}^{(r)} & \hat{q}_{2 m-2}^{(r)} & \cdots & \hat{q}_{m}^{(r)} & \hat{q}_{m-1}^{(r)} & \cdots & \hat{q}_{m-n+1}^{(r)} & \hat{q}_{m-n}^{(r)} \\
\hat{p}_{n-m}^{(r)} & \hat{p}_{n-m+1}^{(r)} & \cdots & \hat{p}_{n-1}^{(r)} & \hat{p}_{n}^{(r)} & \cdots & \hat{p}_{2 n-2}^{(r)} & \hat{p}_{2 n-1}^{(r)} \\
\vdots & \vdots & \ddots & \vdots & \vdots & \ddots & \vdots & \vdots \\
\hat{p}_{-n-m+4}^{(r)} & \hat{p}_{-n-m+5}^{(r)} & \cdots & \hat{p}_{-n+3}^{(r)} & \hat{p}_{-n+4}^{(r)} & \cdots & \hat{p}_{2}^{(r)} & \hat{p}_{3}^{(r)} \\
\hat{p}_{-n-m+2}^{(r)} & \hat{p}_{-n-m+3}^{(r)} & \cdots & \hat{p}_{-n+1}^{(r)} & \hat{p}_{-n+2}^{(r)} & \cdots & \hat{p}_{0}^{(r)} & \hat{p}_{1}^{(r)}
\end{array}\right| .
$$

For $m, n \in \mathbb{Z}_{<0}$, we define $\widehat{R}_{m, n}^{(r)}$ by

$$
\widehat{R}_{m, n}^{(r)}=(-1)^{m(m+1) / 2} \widehat{R}_{-m-1, n}^{(r)}, \quad \widehat{R}_{m, n}^{(r)}=(-1)^{n(n+1) / 2} \widehat{R}_{m,-n-1}^{(r)} .
$$

Moreover, we introduce $S_{m, n}=S_{m, n}(t, s)$ as

$$
\widehat{R}_{m, n}^{(r)}(z)=\widehat{S}_{m, n}(t, s), \quad z=\frac{t}{2}, \quad r=2 s-m+n .
$$

Then, $\varphi_{i}=\varphi_{i}(t, s)$ given by

$$
\begin{aligned}
\varphi_{0} & =\frac{\sqrt{t}}{2} \frac{\widehat{S}_{m, n}(t, s) \widehat{S}_{m-1, n-1}(t, s-1)}{\widehat{S}_{m, n-1}(t, s) \widehat{S}_{m-1, n}(t, s-1)}, \\
\varphi_{1} & =\frac{\sqrt{t}}{2} \frac{\widehat{S}_{m-1, n}(t, s-1) \widehat{S}_{m, n-1}(t, s+1)}{\widehat{S}_{m, n}(t, s) \widehat{S}_{m-1, n-1}(t, s)}, \\
\varphi_{2} & =\frac{\sqrt{t}}{2} \frac{\widehat{S}_{m-1, n-1}(t, s) \widehat{S}_{m, n}(t, s-1)}{\widehat{S}_{m-1, n}(t, s-1) \widehat{S}_{m, n-1}(t, s)}, \\
\varphi_{3} & =\frac{\sqrt{t}}{2} \frac{\widehat{S}_{m, n-1}(t, s) \widehat{S}_{m-1, n}(t, s)}{\widehat{S}_{m-1, n-1}(t, s) \widehat{S}_{m, n}(t, s)}
\end{aligned}
$$

solve the symmetric form of $\mathrm{P}_{\mathrm{V}}(1.10)$ for the parameters

$$
\left(\alpha_{0}, \alpha_{1}, \alpha_{2}, \alpha_{3}\right)=\left(\frac{1}{2}-s-n, s, \frac{1}{2}-s+m, s-m+n\right) .
$$


Let us consider the continuum limit of the result in Theorem 2.3. First, we note that the continuous $q$-Laguerre polynomials $P_{k}^{(\alpha)}(y \mid q)$ are expressed as

$$
\begin{array}{r}
P_{k}^{(\alpha)}(y \mid q)=\frac{\left(q^{\alpha+1} ; q\right)_{k}}{(q ; q)_{k}}{ }_{3} \phi_{2}\left(\begin{array}{r}
q^{-k}, q^{\frac{1}{2} \alpha+\frac{1}{4}} e^{i \theta}, q^{\frac{1}{2} \alpha+\frac{1}{4}} e^{-i \theta} \\
q^{\alpha+1}, 0
\end{array} \mid q ; q\right) \\
y=\cos \theta \text { for } k \geq 0
\end{array}
$$

in terms of the basic hypergeometric functions [6]. Then, from (2.2), (2.6) and $(2.7)$, the polynomials $p_{k}^{(b)}(y \mid q)$ are written as

$$
p_{k}^{(b)}(y \mid q)=\left(q^{\frac{3}{4}} b^{-1}\right)^{k} \frac{\left(b^{2} ; q\right)_{k}}{(q ; q)_{k}} 3 \phi_{2}\left(\begin{array}{c}
q^{-k},-q^{-\frac{1}{2}} b x,-b x^{-1} \\
b^{2}, 0
\end{array} \mid q ; q\right) \quad \text { for } k \geq 0
$$

Setting

$$
b=q^{\frac{r}{2}}, \quad q=e^{\frac{\varepsilon^{2}}{2}}, \quad x=-e^{\frac{\varepsilon}{2} \sqrt{t}},
$$

and taking the limit as $\varepsilon \rightarrow 0$, we obtain

$$
\lim _{\varepsilon \rightarrow 0} p_{k}^{(b)}(y \mid q)=\frac{(r)_{k}}{k !}{ }_{1} F_{1}\left(\begin{array}{c|c}
-k & \frac{t}{2}
\end{array}\right)=L_{k}^{(r-1)}(t / 2) \quad \text { for } k \geq 0 .
$$

Similarly, we see that $q_{k}^{(b)}$ reduce to $L_{k}^{(r-1)}(-t / 2)$. Thus, we get

$$
\lim _{\varepsilon \rightarrow 0} R_{m, n}^{(b)}(y \mid q)=\widehat{R}_{m, n}^{(r)}(z),
$$

and

$$
\lim _{\varepsilon \rightarrow 0} S_{m, n}(x, a)=\widehat{S}_{m, n}(t, s)
$$

with $a=q^{s}$. Finally, setting as

$$
f_{i}=-e^{-\varepsilon \varphi_{i}},
$$

we find that (2.10) and (2.9) reduce to (C.5) and (C.6), respectively. It is shown that $q-\mathrm{P}_{\mathrm{V}}(1.1)$ reduce to the symmetric form of $\mathrm{P}_{\mathrm{V}}(1.10)$ by the above limiting procedure in Section 1. Therefore, the rational solutions of $q-\mathrm{P}_{\mathrm{V}}$ stated in Theorem 2.3 reduce to those of $\mathrm{P}_{\mathrm{V}}$. 


\section{REFERENCES}

[1] B. Grammaticos and A. Ramani, Discrete Painlevé equations: coalescences, limits and degeneracies, Phyica, A228 (1996), 160-171.

[2] B. Grammaticos, A. Ramani and V. G. Papageorgiou, Do integrable mappings have the Painlevé property, Phys. Rev. Lett., 67 (1991), 1825-1828.

[3] K. Kajiwara, M. Noumi and Y. Yamada, A Study on the fourth q-Painlevé Equation, J. Phys. A: Math. Gen., 34 (2001), 8563-8581.

[4] K. Kajiwara, M. Noumi and Y. Yamada, Discrete dynamical systems with $W\left(A_{m-1}^{(1)} \times A_{n-1}^{(1)}\right)$ symmetry, Lett. Math. Phys., 60 (2002), 211-219.

[5] K. Kajiwara, M. Noumi and Y. Yamada, private communication.

[6] R. Koekoek and R. F. Swarttouw, The Askey-scheme of hypergeometric orthogonal polynomials and its q-analogue, Delft University of Technology, Department of Technical Mathematics and Informatics Report no. 98-17 (1998).

[7] K. Koike, On the decomposition of tensor products of the representations of the classical groups: by means of the universal characters, Adv. Math., 74 (1989), 57-86.

[8] T. Masuda, Y. Ohta and K. Kajiwara, A determinant formula for a class of rational solutions of Painlevé V equation, Nagoya Math. J., 168 (2002), 1-25.

[9] M. Noumi, S. Okada, K. Okamoto, and H. Umemura, Special polynomials associated with the Painleve equations II, Proceedings of the Taniguchi Symposium, 1997, Integrable Systems and Algebraic Geometry (M. H. Saito, Y. Shimizu, K. Ueno, eds.), Singapore: World Scientific (1998), pp. 349-372.

[10] M. Noumi and Y. Yamada, Higher order Painlevé equations of type $A_{l}^{(1)}$, Funkcial. Ekvac., 41 (1998), 483-503.

[11] K. Okamoto, Studies on the Painlevé equations III, second and fourth Painlevé equations, $\mathrm{P}_{\mathrm{II}}$ and $\mathrm{P}_{\mathrm{IV}}$, Math. Ann., 275 (1986), 222-254.

[12] A. Ramani, B. Grammaticos and J. Hietarinta, Discrete versions of the Painlevé equations, Phys. Rev. Lett., 67 (1991), 1829-1832.

[13] H. Sakai, Rational surfaces associated with affine root systems and geometry of the Painlevé equations, Commun. Math. Phys., 220 (2001), no. 1, 165-229.

[14] T. Tokihiro, D. Takahashi, J. Matsukidaira and J. Satsuma, From soliton equations to integrable cellular automata through a limiting procedure, Phys. Rev. Lett., 76 (1996), 3247-3250.

[15] M. Taneda, Polynomials associated with an algebraic solution of the sixth Painlevé equation, to appear in Jap. J. Math., 27 (2001).

[16] H. Umemura, Special polynomials associated with the Painlevé equations I, preprint.

Department of Mathematics

Kobe University

Rokko

Kobe, $657-8501$

Japan

masuda@math.kobe-u.ac.jp 